\title{
Distinct influence of different vascular risk factors on white matter brain lesions in multiple sclerosis
}

\author{
Ruth Geraldes (D) , ${ }^{1,2}$ Maciej Juryńczyk ${ }^{1}$ Giordani dos Passos, ${ }^{1}$ Alexander Prichler, ${ }^{3}$ \\ Karen Chung, ${ }^{4}$ Marloes Hagens, ${ }^{5}$ Serena Ruggieri, ${ }^{6}$ Elena Huerga, ${ }^{7}$ \\ Jaume Sastre-Garriga, ${ }_{1}^{8}$ Christian Enzinger, ${ }^{3}$ Declan T Chard, ${ }_{1}^{4,9}$ Frederik Barkhof, ${ }_{1}^{10,11}$ \\ Claudio Gasperini, ${ }^{6}$ Alex Rovira, ${ }^{12}$ Gabriele C DeLuca, ${ }^{1}$ Jacqueline Palace, ${ }^{13}$ on behalf \\ of the MAGNIMS study group
}

\begin{abstract}
- Additional material is published online only. To view please visit the journal online (http://dx.doi.org/10.1136/ jnnp-2019-322369).
\end{abstract}

For numbered affiliations see end of article.

\section{Correspondence to}

Dr Jacqueline Palace, Nuffield Department of Clinical Neurosciences, John Radcliffe Hospital, Oxford OX3 9DU, UK; jacqueline.palace@ndcn.ox. ac.uk

Received 1 November 2019 Revised 10 January 2020 Accepted 15 January 2020

\section{Check for updates}

(C) Author(s) (or their employer(s)) 2020. No commercial re-use. See rights and permissions. Published by BMJ.

\begin{tabular}{l}
\hline To cite: Geraldes R, \\
Juryńczyk M, dos Passos G, \\
et al. J Neurol Neurosurg \\
Psychiatry Epub ahead of \\
print: [please include Day \\
Month Year]. doi:10.1136/ \\
jnnp-2019-322369 \\
\hline
\end{tabular}

\section{ABSTRACT}

Objective To determine if vascular risk factor (VRF), that is, smoking, arterial hypertension (HT), dyslipidaemia and diabetes, have an effect on multiple sclerosis (MS) pathology as measured by MS typical brain lesions, we have compared brain MRIs from patients with MS with and without VRF age-matched and sex-matched.

Methods Brain MRIs from five centres were scored for the presence of Dawson's fingers (DF) and juxtacortical lesions (JCL). A regression model was built to predict the effect of each individual VRF on DF and JCL, considering age and disease duration.

Results $92 \mathrm{MS}$ cases without VRF and $106 \mathrm{MS}$ with one or more VRF (80 ever-smokers, 43 hypertensives, 25 dyslipidaemics and 10 diabetics) were included. Ever-smoking associated with a higher burden of DF $(\operatorname{Exp}(B)=1.29,95 \% \mathrm{Cl} 1.10$ to $1.51, p<0.01)$ and $J C L$ $(\operatorname{Exp}(B)=1.38,95 \% \mathrm{Cl} 1.21$ to $1.57, p<0.01)$. No other VRF had an impact on DF. Dyslipidaemia associated with increased JCL $(\operatorname{Exp}(B)=1.30,95 \% \mathrm{Cl} 1.10$ to $1.56, p<0.01)$ but HT did not associate with any of the outcomes.

Conclusions Individual VRF appear to affect MSspecific lesions differently. An increase in MS lesions was mainly seen in smokers; however, this VRF is most likely to be present from onset of MS, and other VRF effects may be partly mitigated by treatment. Our findings support that treating VRF and cessation of smoking may be important in the management of MS.

\section{INTRODUCTION}

Vascular risk factors (VRF) and comorbidities are associated with worse clinical outcomes in multiple sclerosis (MS). ${ }^{1}$ Higher T2 white matter brain lesions (WML) volumes have been seen in patients with MS with concomitant arterial hypertension (HT) and smoking. ${ }^{2}$ It is not clear whether or not this increase of lesion load is due to a higher burden of MS or vascular WML nor whether the effects of individual VRF on MS WML differ. Several lesion characteristics segregate more clearly with MS, namely juxtacortical U-shaped or S-shaped, ovoid or elongated well-demarcated lesions perpendicular to the wall of lateral ventricle (Dawson's fingers). ${ }^{4}$ In the present study, we aimed to determine if there is an excess of these 'MS-specific' lesions on conventional MRI in MS with VRF compared with
MS without VRF and to explore the effect of each individual VRF on 'MS-specific' lesions.

\section{MATERIAL AND METHODS}

\section{Study design and cohorts}

A multicentre retrospective case-control study was set up to compare MS cases with and without VRF. Anonymised clinical and imaging data were obtained from five MRI in MS (MAGNIMS-www. magnims.eu) network centres: Graz, London, Rome, Barcelona and Amsterdam. Each centre contributed pairs of age-matched and sex-matched MS cases with and without VRF. The imaging and clinical data were collated centrally in Oxford, according to local ethics regulations, quality controlled and blinded for the visual scoring. MS cases were diagnosed according to the $2010 \mathrm{McD}$ onald criteria and exclusion criteria were presence of previous stroke or high risk for cardioembolic stroke (eg, atrial fibrillation, valvular heart disease), other neurological diseases or known non-MS brain lesions.

Demographic and clinical data were provided including: sex, age, disease duration and disease modifying treatment (DMT) at the time of the MRI (yes, no and if latter if they have ever had treatment). The presence or absence of the following VRF was recorded according to previously used definitions: ${ }^{56}$ (1) HT (ever); (2) hypercholesterolemia (ever); (3) diabetes mellitus (DM); (4) self-reported smoking status-positive if patients ever smoked $>10$ cigarettes a day for at least 6 months (table 1). T1 and T2-Fluid Attenuated Inversion Recovery (FLAIR)weighted brain imaging sequences obtained from $3 \mathrm{~T}$ brain MRIs were provided, with variable sequence protocol depending on the source of the data but of established high quality.

\section{Visual scoring}

A single investigator (RG), blinded to the VRF status, performed visual scoring of the brain MRIs. Inter-rater and intrarater variability was tested on a subset $(n=100)$ by repeat blind scoring by RG and two other independent blinded evaluators (MJ, GP). MS-like WML recorded were: (1) the number of Dawson's fingers (defined as well demarcated periventricular lesions, with elongated, ovoid or flame-like shape, perpendicular to the wall of the lateral ventricles), touching the ventricular 
Table 1 Summary of the effect of any VRF (Model 1) and of each individual VRF (Model 2) on the number of Dawson's fingers and JCL

\begin{tabular}{|c|c|c|c|c|c|c|c|c|c|}
\hline \multirow[b]{2}{*}{ Parameter } & \multicolumn{3}{|c|}{ Dawson's fingers } & \multicolumn{3}{|l|}{$\mathrm{JCL}$} & \multicolumn{3}{|l|}{ U-S JCL } \\
\hline & $\operatorname{Exp}(B)$ & $95 \% \mathrm{Cl}, \operatorname{Exp}(\mathrm{B})$ & $P$ value & $\operatorname{Exp}(B)$ & $95 \% \mathrm{Cl}, \operatorname{Exp}(\mathrm{B})$ & $P$ value & $\operatorname{Exp}(B)$ & $95 \% \mathrm{Cl}, \operatorname{Exp}(\mathrm{B})$ & $P$ value \\
\hline \multicolumn{10}{|l|}{ Model 1} \\
\hline $\operatorname{VRF}(n=106)$ & 1.05 & 0.89 to 1.22 & 0.55 & 1.27 & 1.11 to 1.44 & $<0.01^{* *}$ & 0.81 & 0.57 to 1.15 & 0.26 \\
\hline Disease duration & 1.03 & 1.02 to 1.03 & $<0.01^{* *}$ & 1.02 & 1.01 to 1.02 & $<0.01 * *$ & 1.02 & 1.00 to 1.03 & $0.03^{*}$ \\
\hline \multicolumn{10}{|l|}{ Model 2} \\
\hline Smoking ${ }^{*}(\mathrm{n}=80)$ & 1.29 & 1.10 to 1.51 & $<0.01^{* *}$ & 1.38 & 1.21 to 1.57 & $<0.01^{* *}$ & 1.14 & 0.79 to 1.64 & 0.47 \\
\hline Hypertension $(n=43) \dagger$ & 0.86 & 0.70 to 1.106 & 0.17 & 0.85 & 0.71 to 1.01 & 0.06 & 0.61 & 0.36 to 1.02 & 0.06 \\
\hline Dyslipidaemia $(n=25) \ddagger$ & 0.97 & 0.77 to 1.23 & 0.80 & 1.30 & 1.10 to 1.56 & $<0.01$ ** & 0.85 & 0.48 to 1.53 & 0.59 \\
\hline Diabetes $(n=10) \S$ & 0.66 & 0.41 to 1.07 & 0.09 & 0.34 & 0.20 to 0.58 & $<0.01$ ** & 0.35 & 0.09 to 1.46 & 0.15 \\
\hline Disease duration & 1.04 & 1.03 to 1.04 & $<0.01^{* *}$ & 1.02 & 1.02 to 1.03 & $<0.01^{* *}$ & 1.02 & 1.00 to 1.04 & $0.03^{*}$ \\
\hline Age & 0.97 & 0.96 to 98 & $<0.01^{* *}$ & 0.98 & 0.97 to 0.99 & $<0.01^{* *}$ & 0.99 & 0.97 to 1.02 & 0.63 \\
\hline
\end{tabular}

${ }^{* *} \mathrm{P}<0.01$.

${ }^{*}$ Cases were considered to be smokers if they had ever smoked $>10$ cigarettes a day for at least 6 months.

tHypertension was diagnosed if blood pressure $>149 / 90 \mathrm{~mm} \mathrm{Hg}$ or on antihypertensive treatment.

¥Hypercholesterolemia was diagnosed if total cholesterol $>5.0 \mathrm{mmol}(193 \mathrm{mg} / \mathrm{dL}$ ) and/or hypertriglyceridaemia (ever) (triglycerides $>1.8 \mathrm{mmmo} / \mathrm{L}$ (150 $\mathrm{mg} / \mathrm{dL}$ ) or on antidyslipidaemia treatment.

§Diabetes mellitus was diagnosed according to WHO definitions ${ }^{6}$ (diabetes symptoms plus: $\mathrm{HbA}_{1 \mathrm{c}} \geq 6.5 \%$ (48 $\mathrm{mmol} / \mathrm{mol}$ ) or a random venous plasma glucose concentration $\geq 11.1 \mathrm{mmol} / \mathrm{L}$ or a fasting plasma glucose concentration $\geq 7.0 \mathrm{mmol} / \mathrm{L}$ or 2 -hour plasma glucose concentration $\geq 11.1 \mathrm{mmol} / \mathrm{L} 2 \mathrm{hours}$ after $75 \mathrm{~g}$ anhydrous glucose in an oral glucose tolerance test) or on antidiabetic treatment.

$\mathrm{JCL}$, juxtacortical lesions; VRF, vascular risk factor.

lining, on axial T2-FLAIR; (2) number of juxtacortical lesions (well demarcated lesions that touched the cortex on both axial T2-FLAIR and T1); (3) U-shaped or S-shaped juxtacortical lesions-a subset of juxtacortical lesions with $\mathrm{S}$ or U shapes.

\section{Statistical analysis}

Inter-rater agreement was measured by free marginal multirater kappa coefficient. ${ }^{7}$ A Poisson log-linear regression model (Model 1) was used to determine the effect of the presence of any VRF (yes/no) on the number of Dawson's fingers, considering disease duration. The same model was used to assess the relationship of VRF and the number of juxtacortical lesions. A similar regression analysis was repeated but each individual VRF was included in the model (Model 2): ever-smoking, HT, dyslipidaemia and DM, as well as disease duration and age, first to predict the number of Dawson fingers and second the number of juxtacortical lesions. Results are presented as the exponentials of the regression coefficients $(\operatorname{Exp}(B))$ and respective CI. A conservative $\mathrm{p}<0.01$ was used to assess significance. All analyses were performed with SPSS V.25.

Data availability

Raw anonymised data are available for appropriate requests.

\section{RESULTS}

Clinical features of the cohorts used for lesion visual scoring and inter-rater agreement for the visual scoring

Data from $201 \mathrm{MS}$ cases (93 without and 108 with VRF) were available, but three cases were excluded due to the presence of confluent lesions. Clinical features of the cohorts included are depicted in online supplementary table 1 . No significant differences were found between the MS with no VRF and the MS with any VRF regarding age, sex, disease duration, MS subtype or DMT. However, patients with hypertensive $(n=43$, mean age $50.6 \pm 9.3$ years, $\mathrm{p}=0.03)$ and diabetic $(\mathrm{n}=10$, mean age $55.5 \pm 7.8$ years, $\mathrm{p}=0.002)$ MS were significantly older compared with those without VRF ( $n=92,47.5 \pm 8.3$ years).
Substantial inter-rater agreement was found for the number of: Dawson's fingers (0.75), juxtacortical lesions (0.72) and U-S shape juxtacortical $(0.65)$ lesions.

\section{The effects of the presence of VRF on lesions}

The number of juxtacortical lesions was $27.0 \%$ higher in MS with $\operatorname{VRF}(\operatorname{Exp}(\mathrm{B})=1.27,95 \% \mathrm{CI} 1.11$ to $1.44, \mathrm{p}<0.001)$ compared with MS without VRF in a Poisson model adjusting for disease duration (Model 1).

No significant differences between these two groups were found regarding the number of U-shaped or S-shaped juxtacortical lesions or of Dawson's fingers (table 1, Model 1).

\section{The effects of the presence of individual VRF on lesions}

In a model to predict the number of Dawson's fingers, considering each of the VRF and adjusting for disease duration and age (Model 2), ever smoking associated with a higher burden of Dawson's fingers, while no significant association was found with the other VRF (table 1, Model 2).

In a similar model to predict the number of juxtacortical lesions, smoking and dyslipidaemia were associated with a higher burden of juxtacortical lesions and diabetes with a lower number of these lesions. No association was found between HT and juxtacortical lesion number.

None of the individual VRF was associated with the number of U-shaped or S-shaped juxtacortical lesions (table 1, Model 2).

All the studied 'MS-specific' lesions significantly increased with disease duration but interestingly a reverse association was seen with age.

To visually illustrate the effects of smoking alone considering the previous results, we removed patients with smoking plus other VRFs to produce a pure 'MS smokers only' group and a 'MS with non-smoking VRF only' group and compared these groups with 'MS without VRF'. Figure 1 shows that with increased disease duration there is an excess of Dawson's fingers in the 'MS smokers only' group compared with the other two groups, and an increase in juxtacortical lesions in both the 'MS smokers only' compared with the other two groups and 

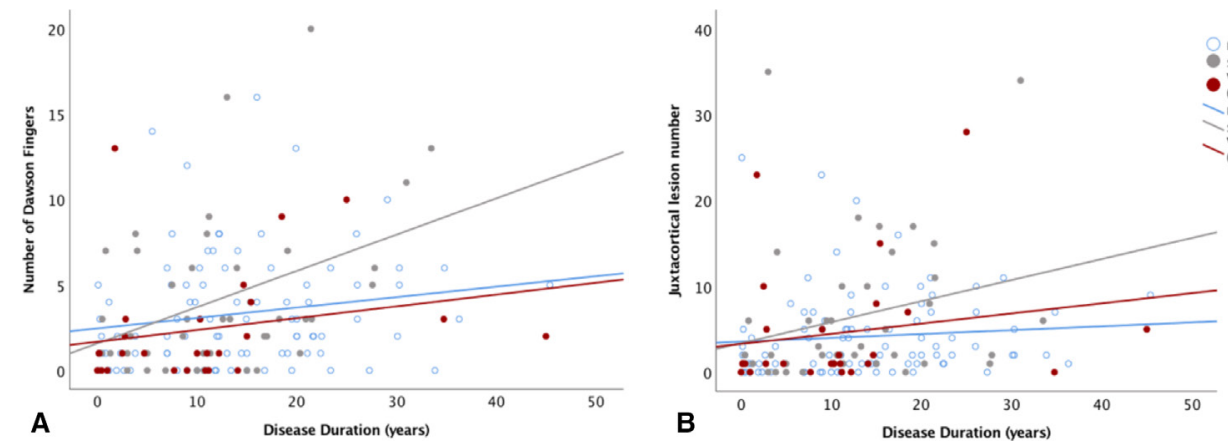

Figure 1 Dawson's fingers (A) and juxtacortical lesion (B) counts against disease duration in MS per VRF group: without VRF (blue line), smoking only (grey line) and VRF other than smoking (red line). MS, multiple sclerosis; VRF, vascular risk factor.

an increase in the 'MS with non-smoking VRF only' group compared with MS without VRF.

\section{Discussion}

This study is novel in studying the effect of different VRF on lesions with features more usually associated with MS, rather than total lesion numbers which does not distinguish between vascular and MS pathology. A key finding of our study is that VRF should not be considered as a composite, as each has a different association with 'MS-specific' lesions. Smoking increased both Dawson's fingers and juxtacortical lesion number, but dyslipidaemia only increased the latter. HT showed no effect on MS-like lesions, while DM was associated with a lower number of juxtacortical lesions.

The documented effect of smoking and hyperlipidaemia on 'MS-specific' lesions supports a direct influence on MS pathology, possibly through promotion of acute inflammation. ${ }^{2-11}$ In line with our results, $\mathrm{HT}^{12}$ and $\mathrm{DM}^{13}$ and total $\mathrm{VRF}^{5}$ have failed to show an impact on T2 lesion volume in MS, although MS-specific lesion locations were not studied, and individual VRFs, including smoking, were not adjusted for. As expected, disease duration had a significant effect on lesions but less expected age had an inverse effect when disease duration was included in the model. This interesting observation is in line with our postmortem studies showing that the number of active plaques decreases with age at death. ${ }^{14} \mathrm{DM}$ and age-related cerebrovascular changes may have an unforeseen negative impact on classic MS WML development.

Our results need to be interpreted with caution since the number of patients included in each individual VRF group was low (from 10 in diabetes group to 80 in the smoking group) and no information regarding VRF treatment that may have an impact on MS WML ${ }^{215}$ was available. Additionally, VRFs may be present for different lengths of time and be managed more or less effectively, for example smoking usually starts in adolescence before the onset of MS and it is not treatable by medication, whereas HT, DM and dyslipidaemia tend to develop later and are treated; thus, smoking may be a higher VRF in our cohort of patients. Finally, our study may have been underpowered to detect an association between the studied VRF and the less commonly found, but possibly more specific, U-shaped or S-shaped juxtacortical lesions.

Despite these limitations, our study highlights that individual VRF should not be grouped together, that they appear to have an effect on MS-like lesions (not just brain lesions per se) and reinforces the importance of supporting patients in giving up smoking. To better understand the effect of each VRF on specific types of WML, future prospective studies matching age across all groups, and risk grading the individual VRFs (eg, pack years for smoking, time and control of individual VRFs) are warranted.

\section{Author affiliations}

${ }^{1}$ Nuffield Department of Clinical Neurosciences, Oxford University Hospitals NHS Foundation Trust, Oxford, UK

${ }^{2}$ Neurology, Frimley Health NHS Foundation Trust, Frimley, UK

${ }^{3}$ Department of Neurology, Medical University of Graz, Graz, Österreich, Austria

${ }^{4}$ NMR Research Unit, Queen Square Multiple Sclerosis Centre, University College London, London, UK

${ }^{5}$ Department of Neurology, MS Center, Vrije Universiteit Amsterdam, Amsterdam, Noord-Holland, The Netherlands

${ }^{6}$ Centro Sclerosi Multipla, Osp. San Camillo Forlanini, Roma, Italy

${ }^{7}$ Magnetic Resonance Unit. Department of Radiology (IDI), Hospital Vall dI'Hebron,

Barcelona, Catalunya, Spain

${ }^{8}$ Multiple Sclerosis Center of Catalonia, Hospital Universitari Vall d'Hebron,

Barcelona, Spain

${ }^{9}$ National Institute for Health Research (NIHR), University College London Hospitals (UCLH) Biomedical Research Centre, London, UK

${ }^{10}$ Department of Radiology, VU Medical Center, MS Center, Amsterdam, Netherlands ${ }^{11}$ Queen Square MS Centre, Department of Neuroinflammation, UCL Institute of Neurology, Faculty of Brain Sciences, University College London, UK; and National Institute for Health Research (NIHR) University College London Hospitals (UCLH) Biomedical Research Centre, London, UK

${ }^{12}$ Unitat de Ressonància Magnètica (IDI), Servei de Radiologia, Vall d'Hebron University Hospital, Barcelona, Spain

${ }^{13}$ Nuffield Department of Clinical Neurosciences, John Radcliffe Hospital, Oxford, UK

Contributors RG and JP: designed and conceptualised study, analysed the data and drafted the manuscript for the intellectual content. MJ, GdP, AP, KC, JS-G, CE, DTC, FB and AR: major role in the acquisition of data and revised the manuscript for intellectual content. MH, SR and EH: major role in the acquisition of data. GCD: interpreted the data and revised the manuscript for intellectual content.

Funding The authors have not declared a specific grant for this research from any funding agency in the public, commercial or not-for-profit sectors.

Competing interests $R G$ has received support for scientific meetings and courses or honoraria for advisory work from Wolfson College, by the EAN, Bayer, Biogen, Merck and Novartis. GdP has received scholarships from the ECTRIMS, the World Federation of Neurology and Novartis; funding for research from Biogen, Novartis and Roche; travel grants from Roche, Sanofi-Genzyme and Teva and fees for editorial content from Bayer, Merck Serono and Roche. KC has received honoraria for speaking at meetings, advisory work or support to attend meetings from Teva, Biogen Idec and Roche. JS-G reports in the last 36 months grants and personal fees from Genzyme, personal fees from: Biogen, Bayer, Merck, Almirall, Bial, Novartis, Roche, TEVA, Celgene; he is Director of Revista de Neurologia, for which he does not receive any compensation, and serves of Editorial Board of Multiple Sclerosis Journal, for which he receives a compensation. CE has received funding for travel and speaker honoraria from Biogen, Bayer, Genzyme, Merck, Novartis, Shire and Teva, research support from Biogen, Merck and Teva, and has served on scientific advisory boards for Bayer, Biogen, Merck, Novartis, Roche and Teva. DTC has received, in the last 3 years, honoraria (paid to his employer) from Excemed for faculty-led education work; had meeting expenses funded by EAN, ECTRIMS, Novartis and Société des Neurosciences. He has received research funding from the International Progressive MS Alliance, the MS society of Great Britain and Northern Ireland and the Institute for Health Research (NIHR), University College London Hospitals (UCLH) Biomedical Research Centre. GCD is supported by the NIHR Biomedical Research Centre (BRC), 
Oxford and has research funding from the Oxford BRC, MRC (UK) and MerckSerono. He has received travel expenses from Bayer Schering, Biogen Idec, Genzyme, Merck Serono and Novartis and honoraria as an invited speaker for Bayer Schering and Novartis. JP is partly funded by highly specialised services to run a national congenital myasthenia service and a neuromyelitis service. She has received support for scientific meetings and honorariums for advisory work from Merck Serono, Biogen Idec, Novartis, Teva, Chugai Pharma and Bayer Schering, Alexion, Roche, Genzyme, Medlmmune, Eurolmmune, MedDay, Abide and ARGENX, and grants from Merck Serono, Novartis, Biogen Idec, Teva, Abide and Bayer Schering. She has received grants from the MS society and Guthie Jackson Foundation for research studies. FB serves as a consultant for Biogen, Bayer, Genzyme, Jansen Research, Merck, Novartis, Roche, Synthon BV and Teva. CG has received compensation for consulting from Bayer and Biogen and speaker's fees for lectures from Biogen, Bayer, Genzyme, Merck, Novartis and Teva. AR serves on scientific advisory boards for Novartis, Sanofi-Genzyme, Icometrix, SyntheticMR, Bayer, Biogen and OLEA Medical and has received speaker honoraria from Bayer, Sanofi-Genzyme, Bracco, MerckSerono, Teva Pharmaceutical Industries Ltd, Novartis, Roche and Biogen.

Patient consent for publication Not required.

Provenance and peer review Not commissioned; externally peer reviewed.

ORCID iD

Ruth Geraldes http://orcid.org/0000-0001-5829-808X

\section{REFERENCES}

1 Marrie RA, Rudick R, Horwitz R, et al. Vascular comorbidity is associated with more rapid disability progression in multiple sclerosis. Neurology 2010;74:1041-7.

2 Geraldes R, Esiri MM, DeLuca GC, et al. Age-related small vessel disease: a potential contributor to neurodegeneration in multiple sclerosis. Brain Pathol 2017;27:707-22.

3 Kappus N, Weinstock-Guttman B, Hagemeier J, et al. Cardiovascular risk factors are associated with increased lesion burden and brain atrophy in multiple sclerosis. $J$ Neurol Neurosurg Psychiatry 2016;87:181-7.
4 Geraldes R, Ciccarelli O, Barkhof F, et al. The current role of MRI in differentiating multiple sclerosis from its imaging mimics. Nat Rev Neurol 2018;14:199-213.

5 Pichler A, Khalil M, Langkammer C, et al. The impact of vascular risk factors on brain volume and lesion load in patients with early multiple sclerosis. Mult Scler 2019;25:48-54.

6 Sacks DB, Arnold M, Bakris GL, et al. Position statement executive summary: guidelines and recommendations for laboratory analysis in the diagnosis and management of diabetes mellitus. Diabetes Care 2011;34:1419-23.

7 Randolph JJ. Free-Marginal Multirater Kappa (multirater K[free]): An Alternative to Fleiss' Fixed-Marginal Multirater Kappa.. In: Joensuu learn Instr Symp, 2005. http:// eric.ed.gov/?id=ED490661

8 Zivadinov R, Weinstock-Guttman B, Hashmi K, et al. Smoking is associated with increased lesion volumes and brain atrophy in multiple sclerosis. Neurology 2009;73:504-10

9 Kowalec K, McKay KA, Patten SB, et al. Comorbidity increases the risk of relapse in multiple sclerosis: a prospective study. Neurology 2017;89:2455-61.

10 Weinstock-Guttman B, Zivadinov R, Mahfooz N, et al. Serum lipid profiles are associated with disability and MRI outcomes in multiple sclerosis. J Neuroinflammation 2011;8:127.

11 Shirani A, Tremlett H. The effect of smoking on the symptoms and progression of multiple sclerosis: a review. J Inflamm Res 2010;3:115-26.

12 Jakimovski D, Gandhi S, Paunkoski I, et al. Hypertension and heart disease are associated with development of brain atrophy in multiple sclerosis: a 5-year longitudinal study. Eur J Neurol 2019;26:87-8

13 Conway DS, Thompson NR, Cohen JA. Influence of hypertension, diabetes, hyperlipidemia, and obstructive lung disease on multiple sclerosis disease course. Mult Scler 2017:23:277-85.

14 Ruth G, Margaret E, Jacqueline P, et al. WED 141 Pathological correlates of age at death in multiple sclerosis. J Neurol Neurosurg Psychiatry 2018;89:A16.

15 Negrotto L, Farez MF, Correale J. Immunologic effects of metformin and pioglitazone treatment on metabolic syndrome and multiple sclerosis. JAMA Neurol 2016;73:520-8. 